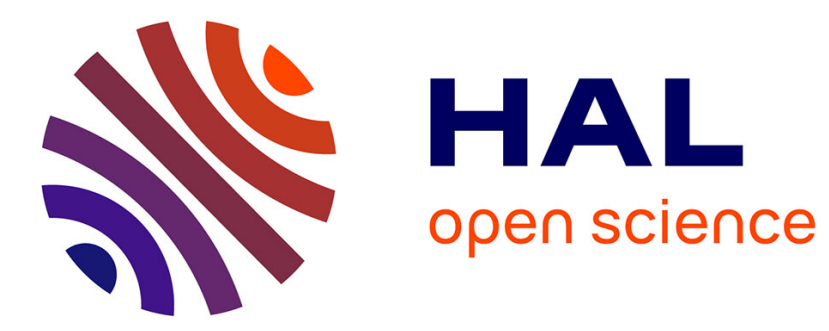

\title{
Reconciling Landmarks and Level Sets
}

Pierre Maurel, Renaud Keriven, Olivier Faugeras

\section{To cite this version:}

Pierre Maurel, Renaud Keriven, Olivier Faugeras. Reconciling Landmarks and Level Sets. ICPR 2006, 18th International Conference on Pattern Recognition, Aug 2006, Hong-Kong, China. pp.69-72, 10.1109/ICPR.2006.979 . inria-00608094

\section{HAL Id: inria-00608094 https://hal.inria.fr/inria-00608094}

Submitted on 12 Jul 2011

HAL is a multi-disciplinary open access archive for the deposit and dissemination of scientific research documents, whether they are published or not. The documents may come from teaching and research institutions in France or abroad, or from public or private research centers.
L'archive ouverte pluridisciplinaire HAL, est destinée au dépôt et à la diffusion de documents scientifiques de niveau recherche, publiés ou non, émanant des établissements d'enseignement et de recherche français ou étrangers, des laboratoires publics ou privés. 


\section{Reconciling Landmarks and Level Sets}

\author{
Pierre Maurel \\ Odyssée Laboratory, ENS \\ Paris, France \\ Pierre.Maurel@ens.fr
}

\author{
Renaud Keriven \\ Odyssée Laboratory, ENPC \\ Marne-la-Valle, France \\ keriven@certis.enpc.fr
}

\author{
Olivier Faugeras \\ Odyssée Laboratory, INRIA \\ Sophia-Antipolis, France \\ Olivier.Faugeras@sophia.inria.fr
}

\begin{abstract}
Shape warping is a key problem in statistical shape analysis. This paper proposes a framework for geometric shape warping based on both shape distances and landmarks. Our method is compatible with implicit representations and a matching between shape surfaces is provided at no additional cost. It is, to our knowledge, the first time that landmarks and shape distances are reconciled in a pure geometric level set framework. The feasibility of the method is demonstrated with two-and three-dimensional examples. Combining shape distance and landmarks, our approach reveals to need only a small number of landmarks to obtain improvements on both warping and matching.
\end{abstract}

\section{Introduction}

Understanding shapes and their basic empirical statistics is a fascinating problem that has attracted the attention of many scientists for many years $[9,8]$. Warping one shape into another is one of the keys leading to statistical shape analysis [6]. Roughly speaking, the warping problem consists in transforming an initial shape into a target one: the result is the family of the intermediate shapes. Slightly different, is the matching problem, where a correspondence between two given shapes has to be established.

Introduced as a way to cope with interface evolution simulation, the level set method $[5,11]$ is based on an implicit representation of surfaces. The natural choice for the implicit representation is often the signed distance function to the closed surface. Consequently, the emergence of shape statistics in the implicit framework is not surprising. The pioneering piece of work considered the distance function as the only object of analysis: warping, matching, or statistical analysis were directly performed on the distance functions $[12,14]$. As we will see in Section 2 and 3 the Hausdorff distance $[4,2]$ can also be used as shape similarity measure.

Though, in the case of complex shapes, providing corresponding landmarks on both the initial and the target shapes reveals to be inevitable The natural way to guide an evolu- tion with landmarks is to try to minimize the distance between the landmarks on the evolving shape and the corresponding ones on the target shape. Again, this yields an irregular motion. In this paper, we present a novel usage of the generalized gradients introduced in [3] that turns this motion into a regular and well posed one.

Remarkably, two recents advances in the level set method make our shape evolution compatible with it: first, a way to simulate a Partial Differential Equation (PDE) embedded on a surface [1], second, a way to deal with surface evolutions involving non normal velocities (and, but this is related, to track the surface points along time) [13].

We first review some shape distances and their usage for the shape warping problem. Then, after introducing the generalized gradient proposed by [3], we present our landmark-guided warping. The next Section discusses the level set implementation of our method. The final Section shows two- and three-dimensional results and comparisons.

\section{Shapes and Shape Metrics}

In our context we define a shape $\Gamma$ to be the boundary of a regular and bounded subset of $\mathbb{R}^{n}$, contained in the image $\Omega$. We denote by $S$ the set of shapes. We refer the reader to [4] for a more rigorous and complete analysis.

In order to compare shapes, a way to quantify the similarity between them must be defined. One of the broadly used distance between shapes is the Hausdorff distance:

$$
d_{H}\left(\Gamma_{1}, \Gamma_{2}\right)=\max \left\{\sup _{\mathbf{x} \in \Gamma_{1}} d_{\Gamma_{2}}(\mathbf{x}), \sup _{\mathbf{x} \in \Gamma_{2}} d_{\Gamma_{1}}(\mathbf{x})\right\}
$$

where $d_{\Gamma}$ is the distance function to the shape $\Gamma$ :

$$
d_{\Gamma}(\mathbf{x})=\inf _{\mathbf{y} \in \Gamma} d(\mathbf{x}, \mathbf{y}) .
$$

The signed distance function to a shape $\Gamma$, denoted by $\tilde{d}_{\Gamma}$, is equal to $d_{\Gamma}$ outside $\Gamma$ and equal to $-d_{\Gamma}$ inside $\Gamma$. An other possible shape distance is then the norm of the Sobolev space, $W^{1,2}(\Omega)$, of square integrable functions with square integrable derivatives:

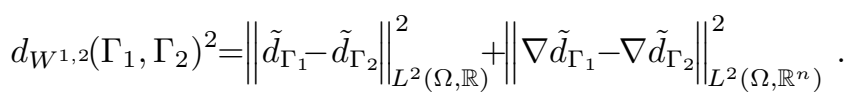




\section{Variational Shape Warping}

In this Section, we review the initial work of [2] and its extension [3].

We assume that we are given a function $E: S \times S \rightarrow$ $\mathbb{R}^{+}$, the energy. This energy can be thought of as a measure of dissimilarity between two shapes. Warping a shape $\Gamma_{1}$ into another one $\Gamma_{2}$ can be stated as the minimization of the energy $E\left(., \Gamma_{2}\right)$ starting from $\Gamma_{1}$, i.e. finding a family of shapes $\{\Gamma(t), t \geq 0\}$ with $\Gamma(0)=\Gamma_{1}$ and $\Gamma(t)$ following some gradient descent toward $\Gamma_{2}$.

\subsection{Shape gradient}

In order to define the gradient of the energy functional, the first step is to compute its Gâteaux derivatives in all directions, i.e. for all admissible velocity fields $\mathbf{v}: \Gamma \rightarrow \mathbb{R}^{n}$. Let us denote by $\mathcal{G}_{\Gamma}\left(E\left(\Gamma, \Gamma_{2}\right), \mathbf{v}\right)$ the Gâteaux derivatives of the energy function $E\left(\Gamma, \Gamma_{2}\right)$ with respect to the shape $\Gamma$ and in the direction $\mathbf{v}$ :

$$
\mathcal{G}_{\Gamma}\left(E\left(\Gamma, \Gamma_{2}\right), \mathbf{v}\right)=\lim _{\varepsilon \rightarrow 0} \frac{E\left(\Gamma+\varepsilon \mathbf{v}, \Gamma_{2}\right)-E\left(\Gamma, \Gamma_{2}\right)}{\varepsilon}
$$

For an inner product $\langle,\rangle_{F}$ equipping the deformation space $F$, there exists a vector $\mathbf{w}$ such that:

$$
\forall \mathbf{v} \in F, \mathcal{G}_{\Gamma}\left(E\left(\Gamma, \Gamma_{2}\right), \mathbf{v}\right)=\langle\mathbf{w} \mid \mathbf{v}\rangle_{F}
$$

We call it the shape gradient of E relative to the inner product $\langle,\rangle_{F}$ and we note it $\mathbf{D}_{\Gamma}^{\left(F,\langle,\rangle_{F}\right)} E\left(\Gamma, \Gamma_{2}\right)$. Usually $F$ is taken as the set $L^{2}\left(\Gamma, \mathbb{R}^{n}\right)$ of the square integrable velocity fields on $\Gamma$, and $\langle,\rangle_{F}$ its associated inner product:

$$
\langle f \mid g\rangle_{L^{2}}=\int_{\Gamma} f(\mathbf{x}) \cdot g(\mathbf{x}) d \Gamma(\mathbf{x}) .
$$

In that case, we will only denote the gradient by $\mathbf{D}_{\Gamma} E\left(\Gamma, \Gamma_{2}\right)$.

Equipped with some shape gradient, we can define the warping of a shape $\Gamma_{1}$ into another one $\Gamma_{2}$ as finding the family $\Gamma(t)$ solution of the following Partial Differential Equation:

$$
\begin{aligned}
\Gamma(0) & =\Gamma_{1} \\
\frac{\partial \Gamma}{\partial t} & =-\mathbf{D}_{\Gamma}^{\left(F,\langle,\rangle_{F}\right)} E\left(\Gamma, \Gamma_{2}\right)
\end{aligned}
$$

Natural candidates for the energy function $E$ are the distances presented in the previous Section. The Hausdorff distance is not Gâteaux differentiable. Yet, this problem can be solved using an smooth approximation of this distance, denoted by $\tilde{d}_{H}\left(\Gamma_{1}, \Gamma_{2}\right)$, which presents the advantage of being differentiable (see [2] for more details).

\subsection{Generalized gradient and spatially co- herent flows}

Although mathematically well justified, the warpings induced by $E=\tilde{d}_{H}$ or $E=d_{W^{1,2}}$ do not reveal to be completely satisfying: the obtained deformations do not seem to be the one a human observer would have chosen. To cope with this, the same authors introduced in [3] a way to favor rigid (translations and rotations) and scaling motions. Their approach consists in changing the inner product used in the definition of the gradient (see [3] for more details). Actually, only global coherent motions are promoted by this new gradient. We will see that the symptom of "unnatural" warping persists in case of complex shapes or shapes related by an articulated motion (see the hands example on Fig. 2).

\section{Landmarks-guided warping}

\subsection{The energy}

Landmarks are then necessary in many cases. Provided by the user (anatomical landmarks), or automatically extracted (geometric landmarks), we assume that we are given $p$ pairs of corresponding points on the initial and on the target shapes, $\left\{\left(\mathbf{x}_{1 i}, \mathbf{x}_{2 i}\right) \in \Gamma_{1} \times \Gamma_{2}, 1 \leq i \leq p\right\}$. We would like to use the information given by theses correspondences to guide the evolution given by equation (1). We do this by adding a landmark term $E_{\mathscr{L}}$ to the energy:

$$
E_{t o t}\left(\Gamma, \Gamma_{2}\right)=E\left(\Gamma, \Gamma_{2}\right)+E_{\mathscr{L}}\left(\Gamma, \Gamma_{2}\right)
$$

During an evolution, while forward correspondences may not exist (formation of shocks), backward correspondences are guaranteed: each point of the evolving interface comes from one point at time 0 (see [13]).

We note $\psi_{t}: \Gamma(t) \rightarrow \Gamma_{1}$ the family of functions giving for each point $\mathbf{x}$ of $\Gamma(t)$ the point $\psi_{t}(\mathbf{x})$ on $\Gamma_{1}$ from which $\mathbf{x}$ comes. Let $\gamma_{i}(t)=\psi_{t}^{-1}\left(\left\{\mathbf{x}_{1 i}\right\}\right)$ be the subset of $\Gamma(t)$ coming from $\mathbf{x}_{1 i}$. Equipped with this correspondence, we are now able to define a landmark-based energy as the sum, for each landmark of $\Gamma_{2}$, of the squared distance between this point and the corresponding set $\gamma_{i}(t)$ :

$$
E_{\mathscr{L}}=\sum_{i} d\left(\mathbf{x}_{2 i}, \gamma_{i}(t)\right)^{2}
$$

with the convention that the distance to an empty set is zero. Note that some landmarks might disappear (shock) or become a continuous infinity of points (rarefaction). Actually, we conjecture that, for reasonable choices of the landmarks, rarefaction does not happen with smooth curves. Yet, depending on the initial energy $E$, there might be some shocks, even with smooth curves.

In the sequel, we will suppose that either an initial landmark $\mathbf{x}_{1 i}$ remains one point $\mathbf{x}_{i}(t)\left(\gamma_{i}(t)=\left\{\mathbf{x}_{i}(t)\right\}\right)$, or it disappears $\left(\gamma_{i}(t)=\emptyset\right)$. Under these hypothesis, the energy can be rewritten in the more classical way:

$$
E_{\mathscr{L}}=\sum_{\left\{i, \gamma_{i}(t) \neq \emptyset\right\}} d\left(\mathbf{x}_{i}(t), \mathbf{x}_{2 i}\right)^{2}
$$

keeping in mind that point $\mathbf{x}_{i}(t)$ come from the backward correspondences $\psi_{t}$. 


\subsection{Adapted gradient}

Formally, the energy given by equation (3) yields Dirac peaks in the expression of the gradient of the energy:

$\mathbf{D}_{\Gamma}^{L^{2}} E_{t o t}(\mathbf{x})=\mathbf{D}_{\Gamma}^{L^{2}} E(\mathbf{x})+\sum_{\left\{i, \gamma_{i}(t) \neq \emptyset\right\}} \delta_{\mathbf{x}_{i}(t)}(\mathbf{x})\left(\mathbf{x}_{i}(t)-\mathbf{x}_{2 i}\right)$

where $\delta_{\mathbf{x}}$ denotes the Dirac function centered at point $\mathbf{x}$. This is indeed not a good candidate for a gradient descent.

The solution here is inspired by [3]. We change the inner product which appears in the definition of the gradient. Let $H^{1}\left(\Gamma, \mathbb{R}^{n}\right)$ be the Sobolev space of square integrable velocity fields with square integrable derivatives. We consider the canonical inner product of $H^{1}\left(\Gamma, \mathbb{R}^{n}\right)$ :

$\langle f \mid g\rangle_{H^{1}}=\int_{\Gamma} f(\mathbf{x}) \cdot g(\mathbf{x}) d \Gamma(\mathbf{x})+\int_{\Gamma} \nabla_{\Gamma} f(\mathbf{x}) \cdot \nabla_{\Gamma} g(\mathbf{x}) d \Gamma(\mathbf{x})$ where $\nabla_{\Gamma} f$ and $\nabla_{\Gamma} g$ are respectively the intrinsic derivatives on $\Gamma$.

Interestingly, the $H^{1}$ gradient can be obtained from the $L^{2}$ gradient by solving an intrinsic heat equation with a data attachment term (see [10] for more details): $\mathbf{D}_{\Gamma}^{H^{1}} E_{t o t}$ is solution of

$$
\Delta_{\Gamma} u=u-\mathbf{D}_{\Gamma}^{L^{2}} E_{t o t}
$$

where $\Delta_{\Gamma}$ denotes the intrinsic Laplacian operator on the surface, often called the Laplace-Beltrami operator. The solution of this equation coincides with:

$\underset{u}{\arg \min } \int_{\Gamma}\left|u(\mathbf{x})-\mathbf{D}_{\Gamma}^{L^{2}} E_{t o t}(\mathbf{x})\right|^{2} d \Gamma(\mathbf{x})+\int_{\Gamma}\left|\nabla_{\Gamma} u(\mathbf{x})\right|^{2} d \Gamma(\mathbf{x})$

and the $H^{1}$ gradient is finally a smoothed version of the $L^{2}$ gradient.

\subsection{Matching}

Let us suppose that the warping process of $\Gamma_{1}$ into $\Gamma_{2}$ has converged. More precisely, we suppose there exists some time $T$ such that $\Gamma(T)$ is very close to $\Gamma_{2}$ (e.g. $\left.E_{t o t}\left(\Gamma(T), \Gamma_{2}\right)<\epsilon^{\prime}\right)$, and a way to assimilate points of $\Gamma_{2}$ to points of $\Gamma(T)$ (e.g. taking the closest point ${ }^{1}$ ). Then, the backward correspondence $\psi_{T}$ supplies a natural matching from $\Gamma_{2}$ to $\Gamma_{1}$. This matching is not one to one if some points of $\Gamma_{1}$ have disappeared during the evolution (shocks).

\section{Level set implementation}

There is no need to introduce the broadly known level set method $[5,11]$. However, implementing our scheme in that framework requires two adaptations of the original method: implementing a PDE on an implicit surface and being able to track points during the evolution.

\footnotetext{
${ }^{1}$ This could be a problem if the evolution gets stuck into some local minimum. Yet, we have never experienced this case.
}

\section{1. $H^{1}$ gradient}

The $H^{1}$ gradient, solution of (4), is obtained from an iterative minimization induced by (5). Since the work introduced in [1], implementing a PDE on a surface is affordable in the implicit framework. The only hard point in our case could be the Dirac peaks in the data term. We indeed use a smooth approximation of them.

It should also be mentioned that, in the two dimensional case, the explicit solution of the equation $\Delta_{\Gamma} u=u-v$ is known (see [10] for more details) and can be used to avoid the iterative minimization giving $u$.

\subsection{Point Correspondences}

Because it codes interfaces with implicit representations, the original level set method can not follow the evolution of each point of the initial interface. Only the geometric location of the whole interface is recovered. Then, considered velocities are usually normal to the interface.

In our case, we need to follow the landmark points through the backward correspondences $\psi_{t}$ and to cope with the non normal velocity $-\mathbf{D}_{\Gamma}^{H^{1}} E_{t o t}$. We used a method proposed in [13] which enable to maintain an explicit backward correspondence from the evolving surface to the initial one.

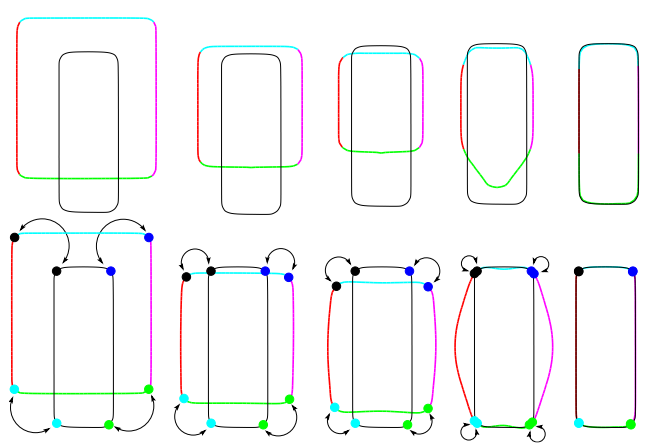

Figure 1. Warping of a rectangle shape into another one. Top row: evolution with $E=$ $d_{W^{1,2}}$. Bottom row: same energy, with four provided landmarks, marked by color spots.

\section{Experiments}

In the experiments showed here, we used the original energy $E=d_{W^{1,2}}$ and test how our landmark-guided force modifies the warping and the final matching. Fig. 1 shows the warping of a rectangle into another one. The different parts of the curves are shown with different colors, so that their respective evolution can be followed. The initial warping without any landmark seems natural but it fails discovering the matching between the edges of the rectangles, a matching indeed recovered when providing landmarks. 
Fig. 2 shows the warping between two hand shapes. The energy $E=d_{W^{1,2}}$ yields an unnatural warping. Adding spatially coherent flows makes the warping a bit better but still fails in some parts. With three landmarks only, both a satisfying warping and a good matching are recovered. Fig. 3 shows the warping of a teddy bear into a cartoon character. Without any landmarks, the top row evolution fails matching the ears and arms of the characters. The bottom row shows the evolution with four landmarks. Red spots allow to check a good matching between landmarks.

\section{Conclusion}

We propose a framework for shape warping based on both shape distances and landmarks. Our method is purely geometric and no extrinsic quantity like a space diffeomorphism has to be considered. Thanks to recent advances in the level set techniques, a level set implementation is possible, reconciling landmarks and the level set methods. Moreover, a matching between shapes is provided at no additional cost. Two- and three-dimensional examples, combining shape distance and landmarks, demonstrate the improvement brought by our approach on both warping and matching, even with a small number of landmarks. Further work includes investigating for a one-to-one matching between shapes, and a way to cope with other landmarks, such as curves on surfaces in $\mathbb{R}^{3}$.

\section{References}

[1] M. Bertalmio, G. Sapiro, L. Cheng, and S. Osher. Variational problems and PDE's on implicit surfaces. In IEEE, editor, IEEE Workshop on Variational and Level Set Methods, pages 186-193, Vancouver, Canada, July 2001.

[2] G. Charpiat, O. Faugeras, and R. Keriven. Approximations of shape metrics and application to shape warping and empirical shape statistics. Foundations of Computational Mathematics, 5(1):1-58, Feb. 2005.

[3] G. Charpiat, R. Keriven, J. Pons, and O. Faugeras. Designing spatially coherent minimizing flows for variational problems based on active contours. In 10th International Conference on Computer Vision, Beijing, China, 2005.

[4] M. Delfour and J.-P. Zolésio. Shape analysis via distance functions: Local theory. In Boundaries, interfaces and transitions, volume 13 of CRM Proc. Lecture Notes, pages 91123. AMS, Providence, RI, 1998.

[5] A. Dervieux and F. Thomasset. A finite element method for the simulation of Rayleigh-Taylor instability. Lecture Notes in Mathematics, 771:145-159, 1979.

[6] I. Dryden and K. Mardia. Statistical Shape Analysis. John Wiley \& Son, 1998.

[7] A. Heyden, G. Sparr, M. Nielsen, and P. Johansen, editors. Proceedings of the 7th European Conference on Computer Vision, Copenhagen, Denmark, May 2002. Springer-Verlag.
[8] D. Kendall. A survey of the statistical theory of shape. Statist. Sci., 4(2):87-120, 1989.

[9] G. Matheron. Random Sets and Integral Geometry. John Wiley \& Sons, 1975.

[10] P. Maurel, R. Keriven, and O. Faugeras. Reconciling landmarks and level sets. Technical Report 5726, INRIA, Oct. 2005.

[11] S. Osher and J. Sethian. Fronts propagating with curvaturedependent speed: Algorithms based on Hamilton-Jacobi formulations. Journal of Comp. Physics, 79(1):12-49, 1988.

[12] N. Paragios, M. Rousson, and V. Ramesh. Matching distance functions: A shape-to-area variational approach for globalto-local registration. In Heyden et al. [7], pages 775-789.

[13] J.-P. Pons, G. Hermosillo, R. Keriven, and O. Faugeras. How to deal with point correspondences and tangential velocities in the level set framework. In International Conference on Computer Vision, volume 2, pages 894-899, 2003.

[14] S. Soatto and A. Yezzi. DEFORMOTION, deforming motion, shape average and the joint registration and segmentation of images. In Heyden et al. [7], pages 32-47.

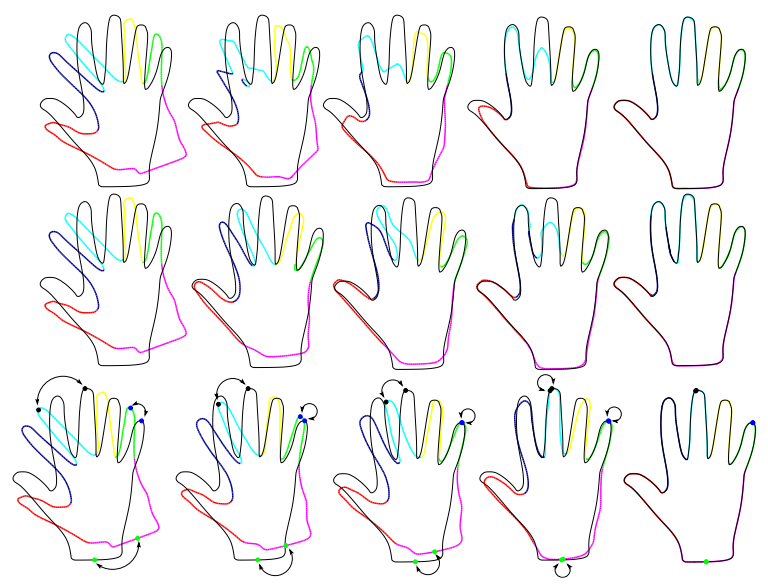

Figure 2. Warping of a hand shape into another one. Top row: evolution with $E=d_{W^{1,2}}$. Middle row: same energy + spatially coherent flows. Bottom row: same energy + spatially coherent flows + three provided landmarks.
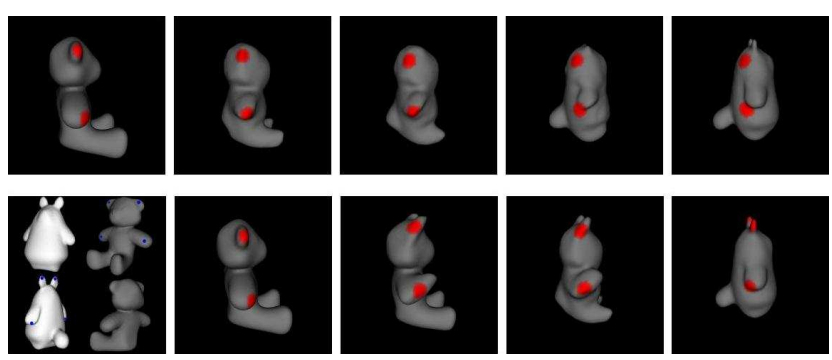

Figure 3. Warping of a teddy bear into a cartoon character. Top row: evolution with $E=$ $d_{W^{1,2}}$. Bottom row, first image: four landmarks (in blue) provided on the two shapes. Bottom row, remaining images: evolution with $E=d_{W^{1,2}}$ plus the provided landmarks. 